\title{
A novel one-sided diffusivity evaluation technique versus Parker's method in application to carbon/epoxy composite
}

\author{
S. S. Pawar*, V. P. Vavilov*
}

* National Research Tomsk Polytechnic University, Savinykh St., 7, Tomsk, 634028 Russia, sspawar@ncsu.edu

\begin{abstract}
Infrared thermography is a fast and contactless inspection technique which is widely used to measure diffusivity as well as to detect impact damage in aerospace composites. Unlike metals, low velocity impact produces extended damage on the rear surface of composite parts thereby posing a significant threat to structural integrity. Front-face thermographic methods are of vital importance in cases where the rear surface is inaccessible for application of conventional transmission techniques. In this paper, we present two different techniques, namely, laser flash intransmission method and recently introduced one-sided thermographic method, to determine thermal diffusivity and characterize impact damage in carbon/epoxy composite.
\end{abstract}

\section{Introduction}

The determination of thermo-physical properties is essential for the specification, development and quality control of materials. The research in various industrial fields [1-6] is more involved in the determination of diffusivity than any other thermo-physical parameter as it provides direct and indirect information about materials used in these fields. Direct information is useful in many fields e.g. in aircraft and power production industries, for the selection of best insulating coating to increase the efficiency of gas turbine and to monitor ageing of such coatings [1]; in aeronautical and automobile industries, for the selection of best suited Carbon/Carbon (C/C) composites for high performance brakes [2]. Indirect information is usually obtained by thermal analysis, which is of great importance for material characterization. In aerospace industry, this direct and indirect information is used for various purposes like porosity measurement of composites that are used for cryogenic fuel tanks and feedlines [3], to correlate diffusivity with carbon fiber content in case of carbon/epoxy composites [4], characterization of impact damage and fatigue induced damage in composite materials $[5,6]$ etc. Aviation industry demands porosity level lower than $2.5 \%$ for structural parts in most of the cases, as porosity reduces interlaminar shear strength [7] in case of carbon/epoxy composites. A low velocity impact damage poses a great threat to aerospace structures as it can grow significantly due to fatigue. The determination of diffusivity of aerospace composites may help to predict their residual life and prevent potential threats to the structure, as diffusivity is directly related to such threats.

Thermal diffusivity measurements have advantage over stationary techniques used for determining thermal conductivity as they are less time consuming, require shorter heat flux measurement, comparatively easy to control and more accurate. Moreover, thermal diffusivity measurements require smaller sample dimensions and can operate in a wider temperature range - from very low temperatures up to $3000^{\circ} \mathrm{C}$ [8]. Photothermal and photoacoustic techniques [8] have been extensively used for the thermal diffusivity determination in the past. However, the conventional diffusivity determination methods require very precisely defined excitation and position of temperature sensors to measure the temperature at one or two points over the surface of the material to be tested. Pulsed thermography, in contrast to conventional methods, performs temperature measurement over the large area of one of the faces of the sample. The temperature measurement accuracy by using thermography is relatively low ( one tenth of a Celsius degree) in each individual point, however, very large number of data points may result in a noise reduction [9].

Thermography has been widely applied to inspect aircraft composites [5, 10, 11]. Low velocity impact damage, caused by a variety of incidences like dropped tools, hail, stones on the runway, minor collisions on the ground etc. [9], is a widely discussed topic in aerospace industry. Such damage is barely visible on the impacted surface and may not be noticed through visual inspection as it begins as an internal delamination or on the non-impacted (rear) side which is not accessible in real life components [8]. The non-accessibility of the rear surface for the inspection restricts materials evaluation to the reflection mode of pulsed thermography. This situation makes it interesting to simultaneously determine the thermal diffusivity and characterize severity of impact damage in the reflection mode.

In this paper, we present two different techniques, namely, laser flash two-sided method, or classical Parker's technique, and recently introduced one-sided thermographic method, to determine thermal diffusivity and characterize impact damage in carbon/epoxy composite. The aim of this work is to evaluate the possibility of a compromise between the accuracy of the laser flash method and practicality of the novel one-sided thermographic method in application to diffusivity measurements and impact damage characterization in the case of carbon/epoxy composite, considering different types of heating.

\section{Brief description of the methods used}

Thermal diffusivity is a thermophysical property that determines a rate of heat diffusion in materials by heat conduction over time. Thermal diffusivity is usually obtained by performing pulsed thermographic measurements in both 
transmission and reflection mode. A brief description of underlying principle and methodology of both the techniques that are used in this study is discussed in this section.

\subsection{Laser Flash Method}

A type of photothermal techniques, 'laser flash method' [11], is a well-established, standard (ASTM E1461) and unsteady state technique for determining thermal diffusivity of solid materials. A thin material, if deposited with a nearly instantaneous pulsed thermal energy on the front surface, heat conduction takes place from the heated front surface to the back surface, causing a temperature decay of the front surface. The temperature increase on the rear surface as a function of time is calculated by using the following well-known expression:

$$
T=\frac{W a}{K L}\left(1+2 \sum_{n=1}^{\infty}(-1)^{n} e^{-n^{2} \pi^{2} F o}\right)
$$

where thickness $L, K$ and $\alpha$ are the plate thickness, conductivity and diffusivity respectively, $W$ is the Dirac pulse energy and $F_{O}=a \tau / L^{2}$ is the Fourier number.

Assuming sample as isotropic and adiabatic, the thermal diffusivity is determined from the shape of the temperature-time curve, e.g. by determining the time, $\tau_{1 / 2}$, to reach half of the maximal temperature increase:

$$
a=0.1388 L^{2} / \tau_{1 / 2}
$$

The time corresponding to different percentage of the maximum temperature rise can also be used to obtain equations analogous to Eq. (2).The absolute values of the absorbed energy, the temperature rise, and the emissivity of the back surface of the sample are not necessary. Various researchers have introduced different theories to correct each ideal condition (isotropic and adiabatic sample, heating time) in Parker's laser flash method. For example, in the case of a long pulse, Ivanov et al. suggested the following modification to Parker's formula [12]:

$$
a=0.1388 L^{2} /\left(\tau_{1 / 2}-0.5 \tau_{h}\right),
$$

where $\tau_{h}$ is the heat pulse duration. The accuracy of such approximate formula was evaluated as $\Delta a / a<\left(\tau_{h} / 2 \tau_{0.5}\right)$; for example, if $\tau_{h}=\tau_{0.5}$, the accuracy decreases as low as $50 \%$.

Thermographic method, which uses flash lamps instead of lasers, as in Parker's method, has gained popularity because of ease of experimentation and availability of entire surface for diffusivity determination. This thermographic method which uses Parker's formula has been applied for diffusivity measurements in this study.

\subsection{Novel one sided diffusivity approach}

The solution for the temperature decay on the heated front surface is the same as given by Eq. (1) excluding the term $-1^{n}$. The solution for the heated front surface decreases monotonically from a maximum value to the ambient temperature. Hence, introducing an inflection point for further data processing is difficult for one-sided, in-reflection diffusivity determination methods. Some methods where material diffusivity was determined in a front-surface procedure using non-linear fitting $[13,14]$ can be found in literature. Vavilov suggested an approach for the determination of diffusivity by analysis of an artificial function which is obtained by multiplying a front-surface temperature evolution by the cubic root of time [15].

Recently, Vavilov et. al. used this function for both diffusivity determination and impact damage characterization in carbon fiber reinforced composites [5]. The concept is to analyse the $T(\tau) \times \tau^{n}$ function by varying $n$ in the range from 0.01 to 0.49 where temporal minimums appear, and optimize $n$ to get the steepest derivative near a minimum point $\left(\tau_{\min }\right)$ for better and accurate determination of diffusivity. The optimised value of $n=0.4$ corresponds to $F o_{\min }=0.2237$, thus giving material diffusivity value as:

$$
a=0.2237 L^{2} / \tau_{\min }
$$




\section{Experimental setup}

The experimental setup, for one-sided (in reflection) and two-sided (in transmission) pulse thermography, used in this study is shown in figure 1. Pulsed thermographic system employing a long-wave (7-13 $\mu \mathrm{m})$ infrared camera, NEC Avio $\mathrm{TH}-9100$, that uses a $320 \times 240$ uncooled microbolometric array detector, was used for the experimental work.

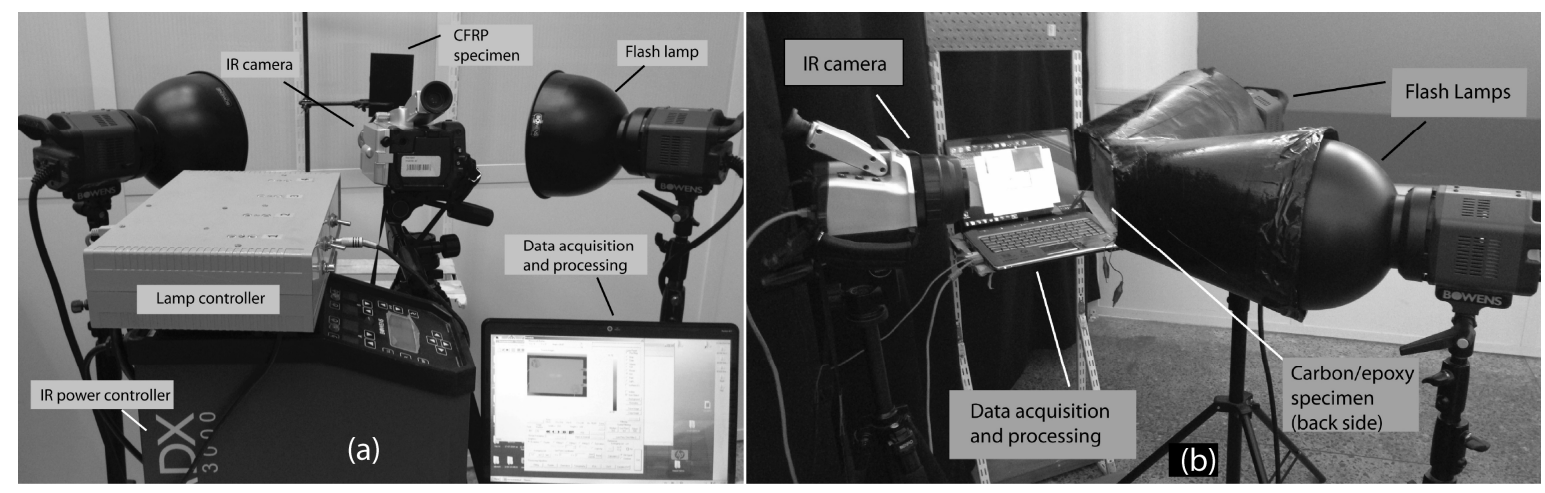

Fig. 1. Flash thermographic NDT experimental set-up for (a) one-sided and (b) two-sided diffusivity measurement

The $3 \mathrm{~mm}$-thick carbon/epoxy specimen which contained the $14 \mathrm{~J}$ impact damage, not visible on the impacted (front) side as shown in figure 2, was used in this study. The specimen was heated by two Xenon lamps, $3.2 \mathrm{~kJ}$ of total energy for flash thermography experiments, and by $1000 \mathrm{~W}$ halogen lamp, for long pulse thermography experiments. Thermal images were acquired on the front (reflection mode) and on the back (transmission mode) side of the specimen at a frame rate of $30 \mathrm{~Hz}$. ThermoFit Pro software was used to process images obtained within the sample heating and cooling stage.
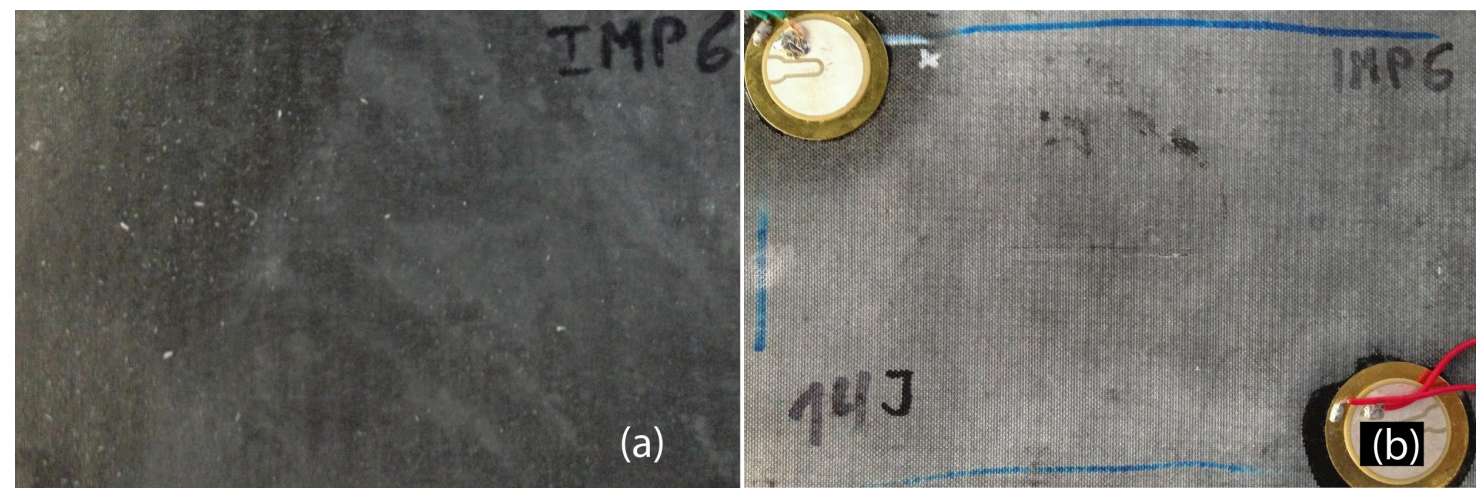

Fig. 2. (a) Front and (b) rear side view of $3 \mathrm{~mm}$ thick carbon/epoxy specimen containing $14 \mathrm{~J}$ of impact damage

\section{Results and discussion}

From recorded thermograms, the thermal diffusivity values were determined by using two different techniques (as mentioned in section 2) and for two different heating conditions to investigate the effect of heating conditions on thermal diffusivity values.

\subsection{Using flash lamps}

Figure 3 shows the temperature rise on the rear surface of the carbon/epoxy specimen, after heating (for $5 \mathrm{~ms}$.) by flash lamps. The temperature rise curve looks as a classical Parker's curve, allowing an easy determination of the diffusivity. The curve half-rise time, $\tau_{1 / 2}$, is 3.01 second that corresponds to $\alpha=4.15 \times 10^{-7} \mathrm{~m}^{2} / \mathrm{s}$. ThermoCalc $2 \mathrm{D}$ software was used to calculate the rear-surface temperature response for the particular sample under non-adiabatic conditions. The diffusivity value which was a solution parameter varied until the simulated curve matched perfectly the experimental curve (figure 3a). The value of diffusivity obtained in this way is $3.97 \times 10^{-7} \mathrm{~m}^{2} / \mathrm{s}$. The values of thermal 
diffusivity obtained by different methods in this study have been compared with the above value as shown in table 1 . In the defective area, the diffusivity value is $2.71 \times 10^{-7} \mathrm{~m}^{2} / \mathrm{s}$ corresponding to half-rise time of 4.61 second (figure $3 \mathrm{~b}$ ).

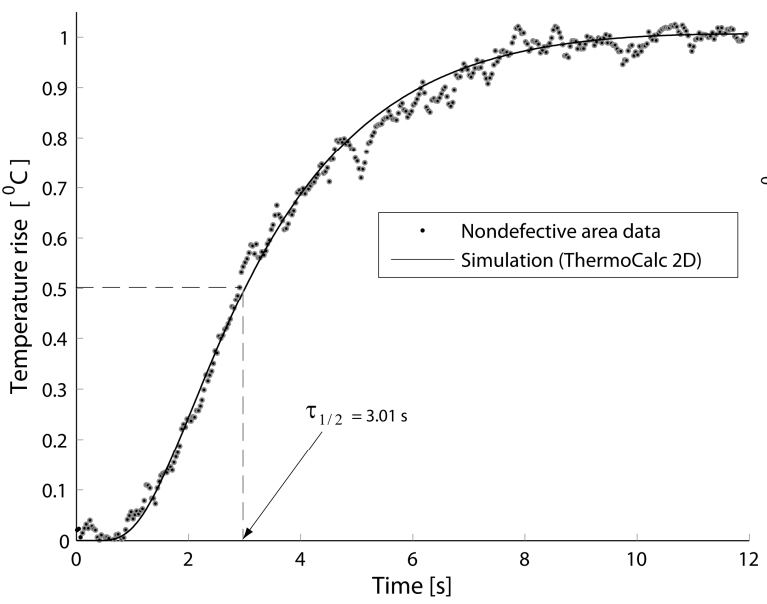

(a)

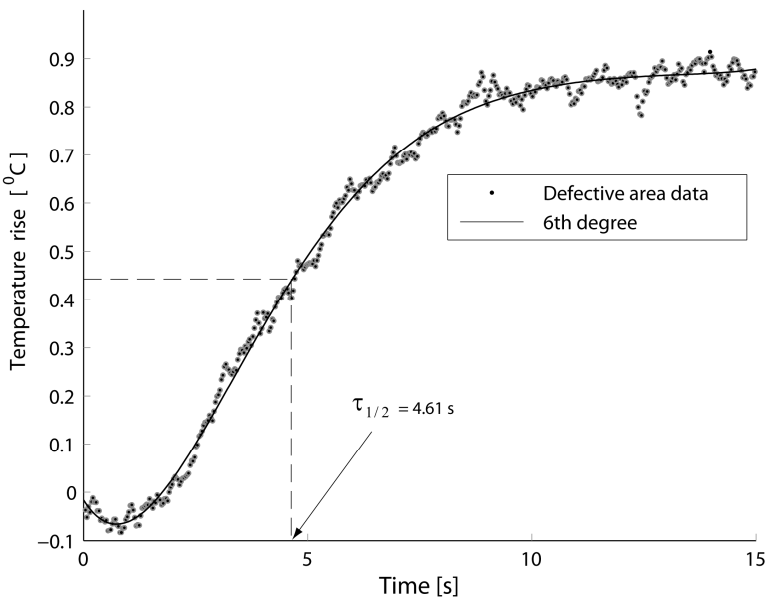

(b)

Fig. 3. Rear surface temperature rise of $3 \mathrm{~mm}$ thick carbon/epoxy specimen under flash heating for diffusivity determination (a) at non-defective area and (b) at defective area, using thermographic flash (two-sided) method

Figure $4 a$ shows the temperature evolution of the front surface after the flash heating, together with the interpolating function that fits the data. Pixel temperatures were smoothed by applying the technique of polynomial fitting. The function $T(\tau) \times \tau^{0.4}$ was then plotted for non-defective area from the front surface. By using the minimum point for this function, thermal diffusivity was calculated by using Eq. (2). The thermal diffusivity value, corresponding to minimum point of 4.79 second is $4.19 \times 10^{-7} \mathrm{~m}^{2} / \mathrm{s}$. Figure $5 \mathrm{a}$ and $\mathrm{b}$, which represent a zoomed version of figure $4 \mathrm{a}$, show the effect of fitting on the accuracy of diffusivity measurement. The change in fitting from polynomial of degree 6 to degree 9 reduces percentage error in diffusivity measurement by $10 \%$.

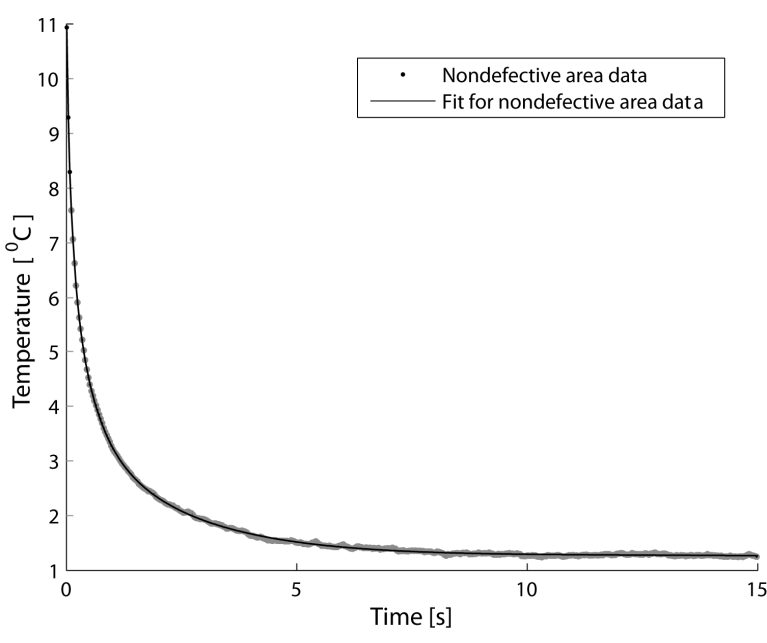

(a)

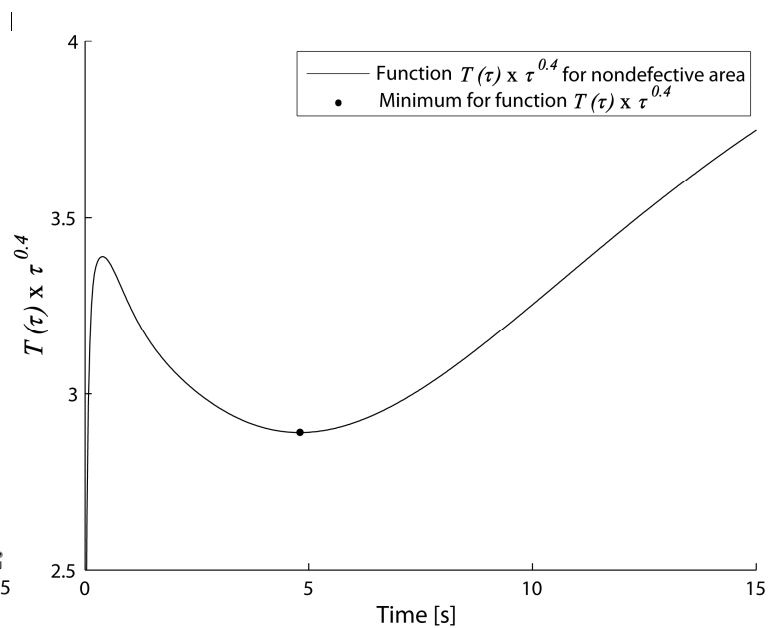

(b)

Fig. 4. (a) Front surface temperature and fit for $3 \mathrm{~mm}$ thick carbon/epoxy specimen under flash heating and (b) diffusivity determination using one sided method 


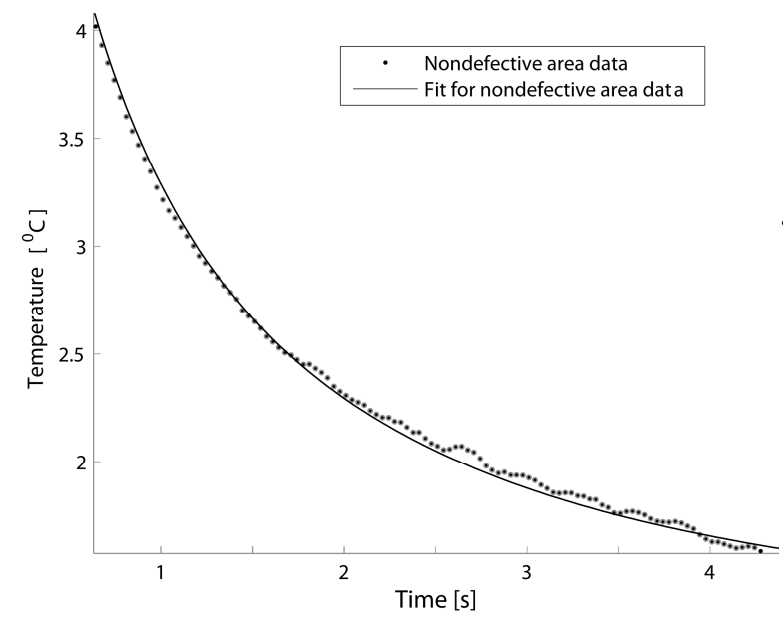

(a)

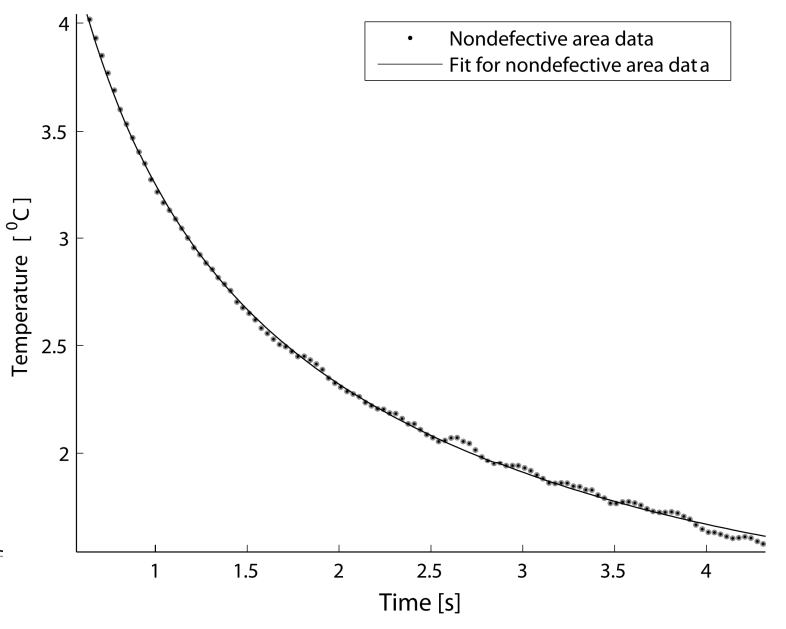

(b)

Fig. 5. Effect of fitting on the accuracy of diffusivity determination using front side method (a) polynomial of $6^{\text {th }}$ degree (b) polynomial of $9^{\text {th }}$ degree

\subsection{Using halogen lamps}

Figure 6 shows the temperature rise on the rear surface of the carbon/epoxy specimen, after 2 seconds of heating with a $1000 \mathrm{~W}$ halogen lamp. The diffusivity value obtained by using Eq. (3) is $\alpha=3.74 \times 10^{-7} \mathrm{~m}^{2} / \mathrm{s}$, corresponding to the curve half-rise time of 4.34 second. The possible decrease in accuracy is because of the tail effect after end of heating and finite pulse duration. The half-rise time for defective area is 6.18 second, thus giving value of $\alpha=2.41 \times 10^{-7}$ $\mathrm{m}^{2} / \mathrm{s}$.

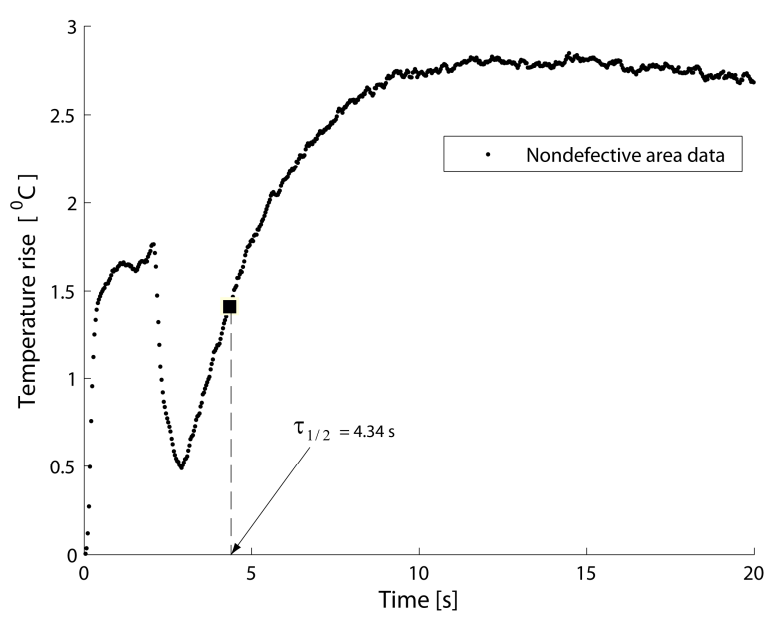

(a)

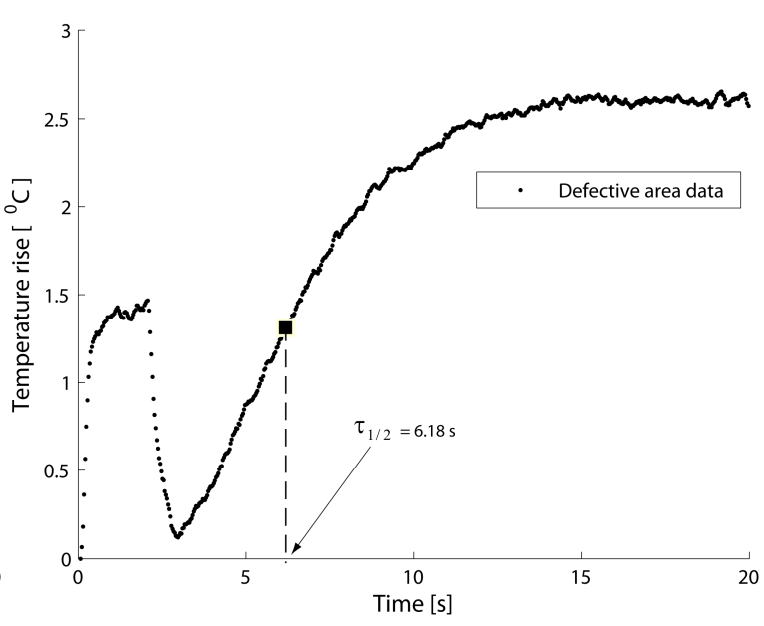

(b)

Fig. 6. Rear surface temperature rise of $3 \mathrm{~mm}$ thick carbon/epoxy specimen under halogen lamp heating for diffusivity determination (a) at non-defective area and (b) at defective area, using thermographic flash (two-sided) method

Figure $7 \mathrm{a}$ shows the temperature evolution and fitted data for the front surface, after long pulse heating. The function $T(\tau) \times \tau^{0.33}$ for non-defective area on the front surface is shown in figure $7 \mathrm{~b}$. By using the minimum point for this function, thermal diffusivity value is obtained as $4.46 \times 10^{-7} \mathrm{~m}^{2} / \mathrm{s}$. From previous results, it is observed that the novel 
method of diffusivity determination works well for flash thermography. In case of long pulse thermography, as can be seen here, it introduces more error. It is believed that, to achieve better accuracy in the case of long-pulse heating, Eq. (4) should be corrected similar to Eq. (3), which is a topic for further study.

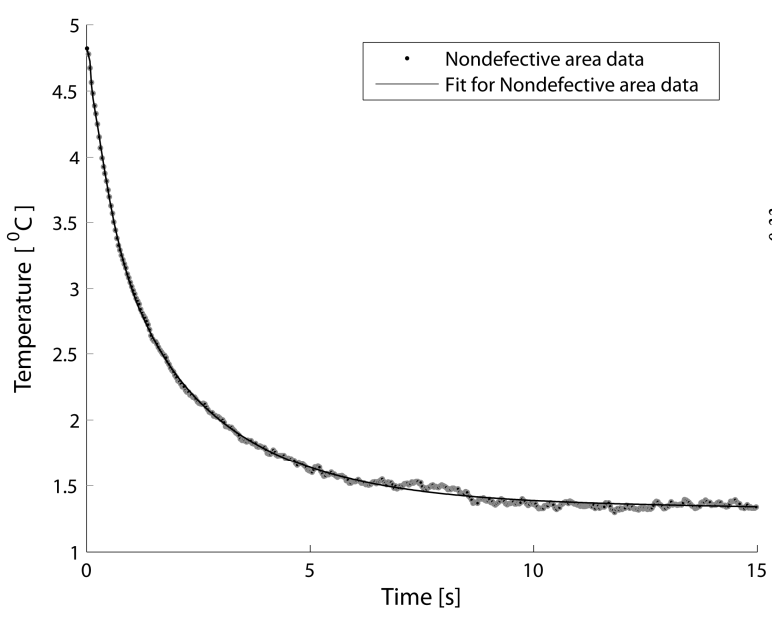

(a)

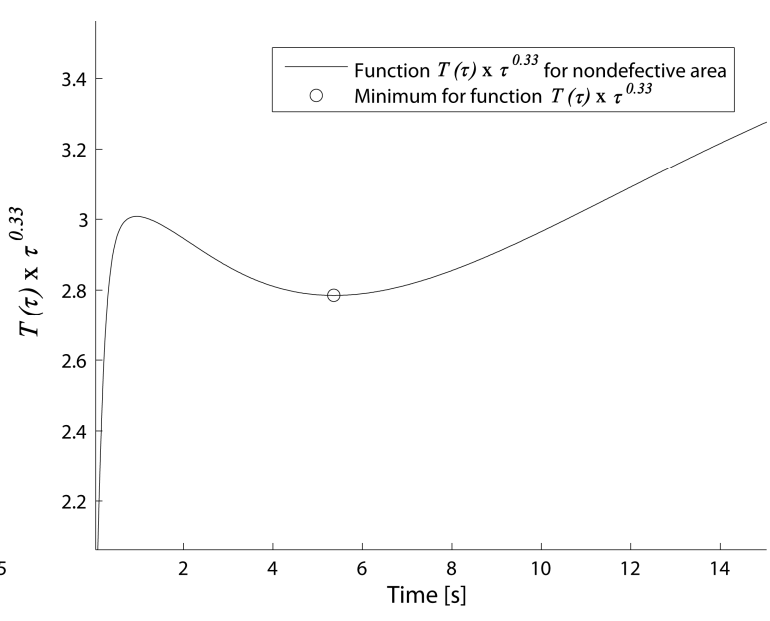

(b)

Fig. 7. (a) Front surface temperature and fit for $3 \mathrm{~mm}$ thick carbon/epoxy specimen under halogen lamp heating and (b) diffusivity determination using one sided method

Table 1. Thermal diffusivity determination of carbon/epoxy composite

\begin{tabular}{|l|c|c|}
\hline Heat source, duration of heating / Technique used & $\begin{array}{c}\text { Thermal } \\
\text { diffusivity } \\
\left(\mathrm{m}^{2} / \mathrm{s}\right)\end{array}$ & $\begin{array}{l}\text { \% error as } \\
\text { compared to } \\
\text { reference } \\
\text { value }\end{array}$ \\
\hline Flash lamps, 5 milliseconds / Transmission (Simulation)
\end{tabular}

${ }^{*}$ Reference value

\subsection{Defect characterization}

Both the methods discussed in this study can be also used for defect characterization. In the case of novel front-face method, the reference-free thermal tomography technique, which was recently applied for impact damage characterization [5], can be used effectively to investigate through-the-thickness damage propagation. In the case of two- 


\section{http://dx.doi.org/10.21611/qirt.2015.0023}

sided testing, 'diffusivity map' can be used for defect characterization. The specimen used for this study did not reveal any defect on the front face during one-sided inspection, therefore, only the results of the two-side testing are discussed.
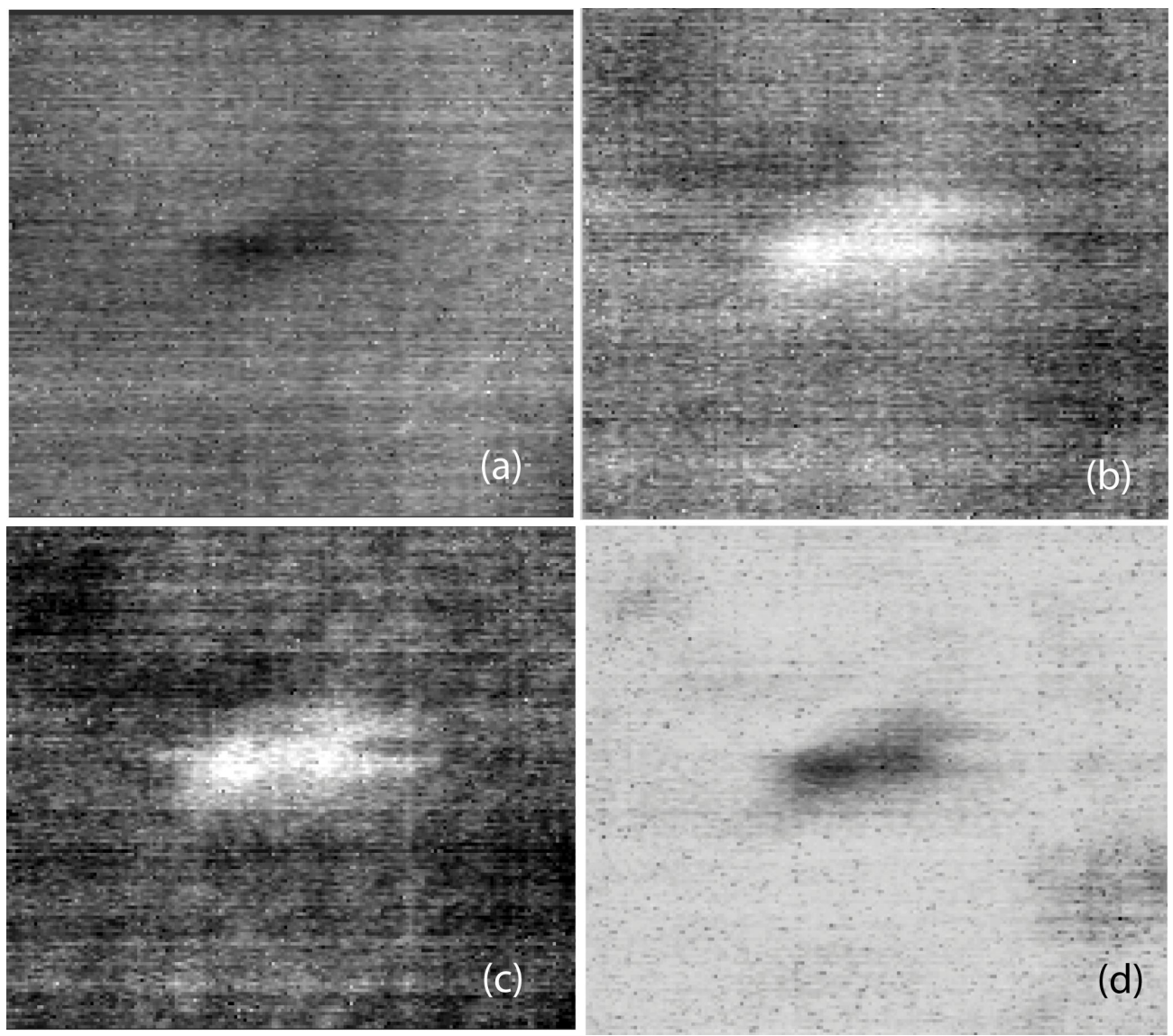

Fig. 8. Qualitative comparison of data processing algorithms in $3 \mathrm{~mm}$-thick carbon/epoxy sample with $14 \mathrm{~J}$ impact damage by using: (a) 'best' IR thermogram, (b) Fourier phasegram, $3^{\text {rd }}$ frequency, (c) Fourier ampligram, and $(d)$ correlation method
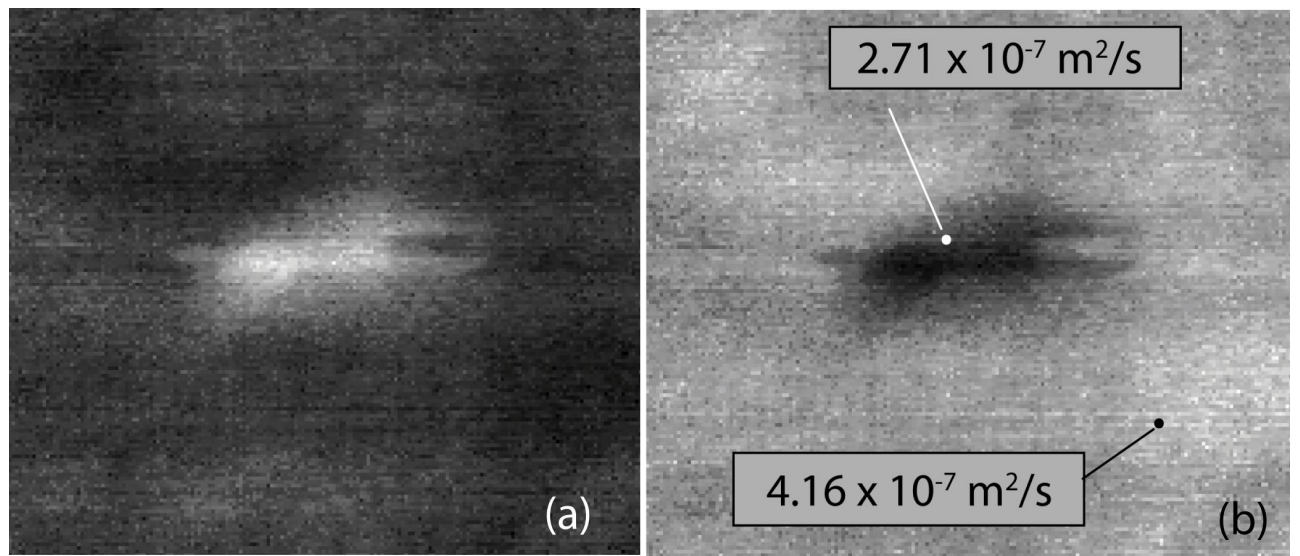

Fig. 9. Determining diffusivity and characterizing impact damage of 14J in 3 mm-thick carbon/epoxy sample: (a) image of $\tau_{1 / 2}$ («timehalfgram»), (b) diffusivity map 
The qualitative comparison of some known data processing algorithms, for experimental results, is illustrated by figure 8 . The best raw thermogram (figure $8 \mathrm{a}$ ) shows a vague indication of the impact damage as the temperature rise on the rear surface was not enough $\left(1.02{ }^{\circ} \mathrm{C}\right)$. Applying the Fourier transform (phase and amplitude image) enhanced the damage indication as shown in figure $8 \mathrm{~b} \& \mathrm{c}$. The correlation algorithm, which is based on the calculation of a correlation coefficient between all pixels and a pixel chosen as a reference, significantly enhanced defect detection by providing a uniform background with the correlation coefficient very close to one and significantly lower at the damage site. Finally, quantitative results are displayed in terms of the time-domain image (timehalfgram) in figure 9a, which seems to be noise-free thus allowing a high-quality map of diffusivity (figure 9b) obtained by Parker's formula. As can be seen from the diffusivity map, in the defective area the diffusivity value drops from $4.16 \times 10^{-7}$ to $2.71 \times 10^{-7} \mathrm{~m}^{2} / \mathrm{s}$. The relative diffusivity variation in this case is $35 \%$ (omitting the negative sign), which is related to the defect severity. As diffusivity maps are synthesized in the time domain, they are quite stable against variations of absorbed energy. It is clearly seen from figure $9 \mathrm{~b}$ that diffusivity maps exhibits fairly uniform distributions in sound areas, which is one of their attractive features.

\section{Conclusions}

In this study, we used two approaches to evaluate thermal diffusivity and characterize low velocity impact damage in carbon/epoxy composite. In non-defect areas, these approaches allowed the determination of material diffusivity in both one- and two-sided test procedures. In defect areas, the so-called diffusivity maps and thermal tomograms may be produced to characterize impact damage. Parker's method proved to be standard and accurate, however, the novel onesided thermographic method, that is convenient from a practical point of view, also demonstrated a reasonable accuracy. Some data processing algorithms being applied to the experimental data improved defect detectability, while a diffusivity map was effectively used to characterize low velocity impact damage.

\section{Acknowledgements}

This research was supported by NIR \#445 (ONG), State order of the Russian Ministry of Higher Education for 20142016. Also, one of the authors (Pawar) would like to thank Tomsk Polytechnic University (TPU) for supporting this study in the framework of the TPU Post-doc program.

\section{REFERENCES}

[1] Bison P.G., Cernuschi F., Grinzato E., Marinetti S., Robba D., "Ageing evaluation of thermal barrier coatings by thermal diffusivity”. Infrared Physics \& Technology, vol. 49, pp. 286-291, 2007.

[2] Pan Y. P., Miller R. A., Chu T. P., and Filip P., "Thermal Diffusivity Measurements and NDE for C/C Composites Using Infrared Thermography". Proceedings of the SEM Annual Conference, 7 pages, 2009.

[3] Chu T. P., Russell S. S. and Walker J. L., "Porosity Measurement in Laminate Composites by Thermography and FEA". NASA Technical Reports Server (NTRS), 4 pages, 2001.

[4] Wróbel G., Rdzawski Z., Muzia G., Pawlak S., "Determination of thermal diffusivity of carbon/epoxy composites with different fiber content using transient thermography". Journal of achievements in materials and manufacturing engineering, vol. 37(2), pp. 518-525, 2009.

[5] Vavilov V. P., Pawar S. S., "A novel approach for one-sided thermal nondestructive testing of composites by using infrared thermography”. Polymer Testing, vol. 44, pp. 224-233, 2015.

[6] Zalameda J. N. et. al., "Thermography Inspection for Early Detection of Composite Damage in Structures during Fatigue Loading". Proc. SPIE 8354, Thermosense: Thermal Infrared Applications XXXIV, 835403, 9 pages, 2012.

[7] Birt E. A. and Smith R. A., "A review of NDE methods for porosity measurement in fibre-reinforced polymer composites". Insight, vol. 46(11), pp. 681-686, 2004.

[8] Cernuschi F. et. al., "Thermal Diffusivity Measurements by Photothermal and Thermographic Techniques". International Journal of Thermophysics, vol. 25(2), pp. 439-457, 2004.

[9] Philippi I., Batsale J. C., Maillet D., and Degiovanni A., "Measurement of thermal diffusivities through processing of infrared images". Review of scientific instruments, vol. 66(1), pp. 182-192, 1995.

[10] Bates D., Smith G., Lu D., Hewitt J., "Rapid thermal non-destructive testing of aircraft components". Composites Part B: Engineering, vol. 31(3), pp. 175-185, 2000.

[11] Parker W.P., Jenkins R.J., Butter C. P., Gutter G. L. and Abbott G.L., "Flash Method of Determining Thermal Diffusivity, Heat Capacity, and Thermal Conductivity". Journal of Applied Physics, vol. 32, pp. 1679-1684, 1961.

[12] Ivanov A. I., Vavilov V.P., "Analysis of pulsed thermal testing procedures". Defectoscopy (Russian Journal of NDT), vol. 1, pp. 77-81, 1987 (in Russian)

[13] Grinzato E., Marinetti S., "Materials NDE by nonlinear filtering applying heat transfer models". Advances in Signal Processing for Non Destructive Evaluation of Materials, NATO ASI Series, Series E: Applied Sciences, 262, pp.117-132, 1994. 
http://dx.doi.org/10.21611/qirt.2015.0023

[14] Zalameda J.N., Winfree W.P., "Improved sampling of thermal transients using Focal Plane Array infrared imagers". Proc. SPIE "Thermosense-XXVI", 5405, pp.374-381, 2004.

[15] Cernuschi F. et. al., "Comparison of thermal diffusivity measurement techniques." QIRT 6th International Conference, 2002. 